\title{
LA DESCENTRALIZACIÓN EN COLOMBIA, ¿REALIDAD O ESPEJISMO? CONSIDERACIONES SOBRE LA ACTUALIDAD DEL PROCESO
}

Decentralization in Colombia: reality or mirage? Considerations on the current process

\section{Jorge Enrique Asela Molina}

Economista por la Universidad Industrial de Santander. Bucaramanga, Colombia. jorgeasela@hotmail.com

\begin{abstract}
Cómo citar / How to cite
Asela, J. (2015). La descentralización en Colombia, ¿realidad o espejismo? Consideraciones sobre la actualidad del proceso. Revista CEA, 1(1), 65-74.
\end{abstract}

Recibido: 22 de abril de 2014

Aceptado: 21 de julio de 2014

\section{Resumen}

En artículo tiene, como primer objetivo, explicar el modelo descentralizado desde un punto de vista teórico, considerado los aportes de diferentes autores de la teoría económica, haciendo principal énfasis en los avances de los aspectos políticos y económicos, en lo correspondiente al manejo del Estado. Como segundo objetivo, se realizará una mirada más cercana al proceso de descentralización en Colombia, partiendo desde la Constitución de 1991, algunos de sus avances, retrocesos y desafíos en la actualidad. Finalmente, se presentará un breve análisis sobre la dependencia de los entes territoriales a los recursos que por transferencias entrega la Nación, esto a través de la descripción del indicador de dependencia intergubernamental planteado por el DNP.

Palabras clave: descentralización política, descentralización fiscal, dependencia.

\begin{abstract}
In this article we will try to explain, as a first objective, the decentralized model from a purely theoretical point of view, considered from the contributions of different authors of economic theory, with special emphasis on the progress of political and economic aspects, concerning the management of the Estate. The second objective of this paper is to take a closer look of the decentralization process in Colombia, starting with the Constitution of 1991, some of its achievements, setbacks and current challenges. Finally, this paper will briefly discuss how much local authorities depend on the funds allocated via transfers done by the Estate, this will be done through the measure description intergovernmental dependence proposed by DNP.
\end{abstract}

Keywords: Political Decentralization, Fiscal Decentralization, Dependency. 


\section{INTRODUCCIÓN}

El proceso secular vivido en Europa generó la pérdida del carácter sacro de las monarquías y su designación divina para gobernar territorios, dando origen al surgimiento de los Estados-nación en el siglo XIX. Es allí donde encuentran su origen los sistemas de organización estatal como estructura fundamental de las nacientes naciones, los modelos centralista y de descentralización emergen como las alternativas a elegir.

Las crisis de endeudamiento económico así como la vuelta de las democracias, tras la caída de las dictaduras militares en las décadas de los setenta y ochenta, pusieron en evidencia en América Latina una lucha entre el modelo centralista y el descentralizado, dicha lucha comenzó a dar un giro hacia el segundo, en lo concerniente a la forma de composición del Estado. El proceso de descentralización trajo consigo ventajas claras, ya que poco a poco -con la evolución de los procesos económicos y el surgimiento de diferentes necesidades en los territorios- fue visto como el más apropiado para dar solución a las necesidades emergentes.

El surgimiento de nuevos servicios públicos, el incremento poblacional, entre otros factores, resultaron claves en la elección de un modelo descentralizado por las naciones. Fundamentos del modelo de descentralización como la participación ciudadana en los procesos de elección de gobernantes y la cercanía de estos con las comunidades, hicieron más eficiente la provisión de bienes y servicios públicos para las localidades, lo que demostró la importancia del proceso a la hora de gobernar y ejecutar los recursos.

Siguiendo este marco, la primera parte del artículo intenta dar un repaso teórico al proceso de descentralización como modelo administrativo de manejo del Estado, mostrando sus aportes a los procesos políticos y económicos. A su vez, se presentan la revelación de preferencias de los individuos como factor importante en el proceso, visto como fundamento de la elección de gobernantes y ejecución de recursos.

En la segunda parte, se intenta dar una mirada al proceso de descentralización colombiano desde la Constitución de 1991, mostrando algunos de los avances, retrocesos y desafíos que este ha enfrentado hasta la actualidad; y finalmente, se presentará un breve análisis sobre la dependencia intergubernamental en el marco de la descentralización fiscal en Colombia, tomando como referencia el indicador de dependencia intergubernamental planteado por el Departamento Nacional de Planeación (DNP).

\section{LA DESCENTRALIZACIÓN POLÍTICO- ADMINISTRATIVA Y EL PAPEL DE LA REVELACIÓN DE PREFERENCIAS}

Se debe entender por descentralización aquel modelo de manejo del Estado, que en las últimas décadas del siglo XX e inicios del siglo XXI, ha tenido mayor correspondencia como forma de plantear el desarrollo desde adentro hacia fuera. Esto realizable a través del plano político y económico.

Prestando atención al plano político, en primera instancia, la descentralización reconoce a los entes subnacionales (término que designa las divisiones territoriales que poseen los Estados soberanos en cualquier nivel, habitualmente con carácter políticoadministrativo) la potestad de crear organismos públicos territoriales que le permitan garantizar la prestación y provisión de bienes y servicios públicos, además de reconocer una autonomía fiscal. 
En el plano económico, esta se enfoca, como bien lo explica Finot, en "trasladar las decisiones sobre asignación de factores de producción desde mecanismos políticoadministrativos hacia la competencia económica» (Finot, 2001, p. 61). Este traslado de decisiones permite a los entes subnacionales minimizar el riesgo proveniente de los vicios del gobierno central tales como la ineficiencia, tamaño, capacidad de reacción; principales generadores de desequilibrios fiscales $y$ sociales.

El proceso de descentralización cuenta con una gran ventaja para los entes subnacionales, ya que como lo mencionamos anteriormente otorga autonomía administrativa y fiscal. Esto puede permitir a los órganos territoriales actuar con mayor prontitud y eficiencia ante las dificultades y requerimientos que se generan en sus territorios.

La cercanía con la comunidad resulta ser el factor clave para que el proceso de descentralización pueda llegar a ser exitoso. Dicha cercanía permite una mejor lectura de las preferencias de los individuos, lo cual garantiza un mejor uso de los recursos y una mejor prestación de bienes y servicios públicos, principal objetivo de la hacienda pública. La garantía depende de factores en los entes territoriales como la capacidad administrativa, técnica e instalada, la cual incluye aspectos como la dotación de recursos físicos, humanos, tecnológicos y maquinaria y equipo; esto en el marco de autonomía y responsabilidad en su gestión.

Surge entonces la disyuntiva entre los individuos a la hora de elegir la localidad donde desean instalarse. Para resolver este dilema, Tiebout (1956) propone que la provisión de bienes y servicios públicos sea el determinante a la hora de tomar esta elección, ya que los individuos se desplazan a las localidades que mejor satisfacen sus requerimientos en cuanto a la prestación de bienes colectivos.

Ahora bien, surge la inquietud, ¿cómo los individuos revelan sus preferencias? Para responder a esa pregunta es importante definir que es un bien colectivo. (Samuelson (1954) define un bien público como aquel que no se encuentra regido por el mercado y cumple con dos características esenciales: (i) la no exclusión y, (ii) la no rivalidad. Estas características hacen referencia, en primer lugar, a que la capacidad de pago de un individuo no puede ser una restricción para adquirirlo; y el segundo, a que la disponibilidad del bien no se ve afectada por el consumo de otra persona.

Otra definición acertada sobre este tipo de bienes es la que otorga Musgrave (1959), el cual divide a los bienes colectivos de dos maneras, las necesidades sociales y los bienes meritorios:

- Las llamadas necesidades sociales tienen como principal característica cumplir el principio de indivisibilidad, lo cual indica que la prestación de estos bienes 0 servicios se hace de manera colectiva, pues no se puede distribuir en porciones precisas e individuales, ejemplo claro de este tipo de bienes y servicios son la justicia y la defensa nacional; además no cumplen con el principio de exclusión. Por último, la revelación de las preferencias de este tipo de bienes no se manifiesta a través del sistema de precios sino por medio de un proceso político.

- A su vez, Richard Musgrave citado por Restrepo (2001) define los bienes meritorios como aquellos que "se consideran tan meritorios que su satisfacción se hace a través del presupuesto público, adicionalmente a lo que se suministra del mismo bien a través del mercado y es pagado por los compradores privados» (2001, p. 28). 
Los bienes meritorios no cumplen con claridad los principios de indivisibilidad y exclusión, un ejemplo claro de este tipo de bien es la educación.

Dadas las características de estos bienes, queda claro que la revelación de las preferencias no se da a través del mercado. Es por esto que Albert Hirschman (1970) propone un modelo que remplaza al mercado en la revelación de las preferencias de los individuos. Este modelo se encuentra fundamentado en la rendición de cuentas por parte de los entes subnacionales, a los individuos. Esto se puede dar a través de dos formas: la primera, por medio de la creación de veedurías ciudadanas; y la segunda, por medio de un mecanismo que Hirschman llama exit, el cual plantea la existencia de más de un proveedor de bienes colectivos, lo que genera cierto grado de competencia y una gama más amplia de escogencia para los consumidores. Es de mencionar que los proveedores de bienes públicos no solo buscan un beneficio de carácter económico, ya que la correcta provisión y prestación de bienes y servicios públicos en la localidad puede generar un beneficio político para su proveedor.

En el mismo sentido -sobre la revelación de las preferencias de los individuos- Oates, citado por Ruiz (2000), plantea que la revelación de las mismas se presentan a través de un proceso político y no de mercado, ya que los consumidores debido a las diferentes restricciones que se generan, no pueden elegir la jurisdicción donde prefieren vivir, pero sí «mediante el proceso político la estructura fiscal y el régimen impositivo y los bienes públicos que prefieren, a partir de la elección sobre un conjunto de gobernantes que constituyen sus posibilidades de consumo» (2000, p. 14).

Al contar con elementos entre los cuales se encuentran: una mayor cercanía con la comunidad, un accionar más ágil a la hora de atender emergencias, el mejor entendimiento de las preferencias de los individuos, un control político más vinculante; hacen que el modelo de descentralización sea el más idóneo como forma de estructurar el Estado.

\section{EL PROCESO DE DESCENTRALIZACIÓN EN COLOMBIA, AVANCES, RETROCESOS Y DESAFÍOS}

A partir de la apertura económica en Colombia, llevada a cabo a inicios de los años noventa, el proceso de descentralización se consolida con la instauración de la Constitución Política de 1991. Esta, en su artículo 1, proclama lo siguiente: «Un Estado social de derecho, organizado en forma de república unitaria, descentralizada, con autonomía de sus entidades territoriales, democrática, participativa y pluralista...» (Constitución Política de Colombia, 1991, p. 3).

En la Constitución se presentan grandes cambios en temas como la orientación política, territorial, distribución de competencias y funciones entre los distintos niveles de gobierno, distribución de recursos entre el gobierno central y lo entes territoriales, procesos de planeación y presupuesto público y sistemas de control fiscal.

Los cambios promovidos en la Constitución de 1991 plantearon nuevos retos para los departamentos y municipios, cambios en las competencias y funciones fueron reglamentados bajo la Ley 60 de 1993, la cual plasmo el rol que los entes territoriales y el gobierno central entrarían a desempeñar dentro del accionar de las políticas públicas. Estas funciones y competencias serán planteadas en la Tabla 1. 
Tabla 1.Competencias y funciones bajo la Ley 60 de 1993. Sectores Sociales.

Table 1. Powers and functions under the Act 60 of 1993. Social Sectors.

\begin{tabular}{|c|c|c|c|}
\hline Sector & Nivel Central & Nivel Departamental & Nivel Municipal \\
\hline $\begin{array}{c}\text { Rol } \\
\text { General }\end{array}$ & $\begin{array}{l}\text { Formular políticas. } \\
\text { Distribuye y transfiere } \\
\text { recursos del situado fiscal. } \\
\text { Administra los fondos de } \\
\text { cofinanciación. } \\
\text { Aconseja a las entidades } \\
\text { territoriales. } \\
\text { Evalúa la prestación de } \\
\text { servicios. }\end{array}$ & $\begin{array}{l}\text { Planea, administra y } \\
\text { distribuye recursos entre los } \\
\text { municipios. } \\
\text { Provee ayuda financiera y } \\
\text { aconseja a los municipios. }\end{array}$ & $\begin{array}{l}\text { Provee los servicios básicos: } \\
\text { salud y educación en el primer } \\
\text { nivel, agua potable y sanidad, } \\
\text { vivienda y otros, que indica la } \\
\text { ley. }\end{array}$ \\
\hline Educación & $\begin{array}{l}\text { Deja de ser responsable de } \\
\text { asuntos administrativos } \\
\text { relacionados nacionales. } \\
\text { educadores nas de } \\
\text { Determina salarios dey } \\
\text { acuerdo con la ley. } \\
\text { Conduce el seguimiento y } \\
\text { los servicios de evaluación. }\end{array}$ & $\begin{array}{l}\text { Planea, administra y } \\
\text { coordina servicios } \\
\text { educativos. } \\
\text { Administra y entrena a los } \\
\text { profesores de acuerdo con } \\
\text { la ley. Transfiere } \\
\text { gradualmente funciones a } \\
\text { los municipios. Administra } \\
\text { fondos de cofinanciación } \\
\text { para los municipios con } \\
\text { recursos locales. }\end{array}$ & $\begin{array}{l}\text { Administra la educación pre- } \\
\text { escolar, primaria y secundaria de } \\
\text { acuerdo con los términos de } \\
\text { cambio departamental. Hace } \\
\text { inversiones necesarias en } \\
\text { infraestructura, equipos y } \\
\text { mantenimiento de centros } \\
\text { educativos. }\end{array}$ \\
\hline Salud & $\begin{array}{l}\text { Promueve y coordina } \\
\text { campañas nacionales y } \\
\text { programas para salud } \\
\text { básica y sanidad. } \\
\begin{array}{l}\text { Desempeña todas las } \\
\text { demás }\end{array} \text { funciones } \\
\text { establecidas por la Ley } 10 \\
\begin{array}{l}\text { de } 1990 . \\
\end{array}\end{array}$ & $\begin{array}{l}\text { Los servicios son prestados a } \\
\text { través de hospitales } \\
\text { regionales especializados y } \\
\text { universitarios. Conduce } \\
\text { campañas en coordinación } \\
\text { gracias al nivel central. } \\
\text { Desempeña todas las demás } \\
\text { funciones establecidas en la } \\
\text { Ley } 10 \text { de } 1990 \text {. }\end{array}$ & 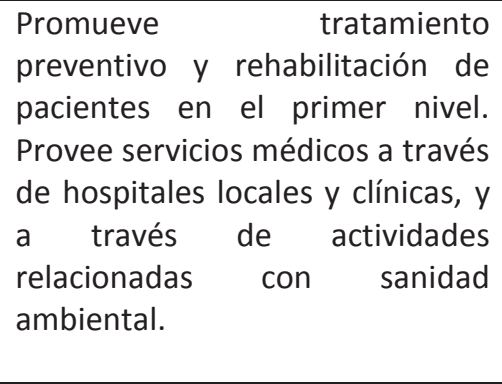 \\
\hline Vivienda & $\begin{array}{l}\text { Promueve ayuda técnica a } \\
\text { entidades locales. } \\
\text { Deja de asumir programas } \\
\text { de construcción. Provee } \\
\text { subsidios a programas } \\
\text { municipales. }\end{array}$ & & $\begin{array}{l}\text { Define la regulación para el } \\
\text { desarrollo urbano. } \\
\text { Promueve y participa en } \\
\text { proyectos de vivienda social. } \\
\text { Provee subsidios a vivienda a los } \\
\text { más necesitados. }\end{array}$ \\
\hline $\begin{array}{l}\text { Sanidad } \\
\text { básica }\end{array}$ & $\begin{array}{lr}\text { Conduce } & \text { programas de } \\
\text { asistencia } & \text { técnica para } \\
\text { entidades } & \text { territoriales. } \\
\text { Cofinancia } & \text { y provee } \\
\text { créditos. } & \end{array}$ & & $\begin{array}{l}\text { Provee servicios (directa o } \\
\text { indirectamente, a través de } \\
\text { contratos con el sector privado). } \\
\text { Conduce proyectos para } \\
\text { construcción y expansión de } \\
\text { acueductos y alcantarillados. } \\
\text { Garantiza la prestación de } \\
\text { servicios básicos rurales de } \\
\text { sanidad, limpieza urbana, } \\
\text { saneamiento de mataderos y } \\
\text { plazas de mercado. }\end{array}$ \\
\hline
\end{tabular}

Fuente: Lleras y Posada. Descentralización política, fiscal y administrativa después de la Constitución de 1991 
A su vez la Constitución define al municipio en el artículo 311 de la siguiente manera:

[El municipio es una] entidad fundamental de la división políticoadministrativa del Estado [que] le corresponde prestar los servicios públicos que determine la ley, construir las obras que demande el progreso local, ordenar el desarrollo de su territorio, la participación comunitaria, el mejoramiento social cultural de sus habitantes y cumplir las demás funciones que le asignen la Constitución y las leyes (Constitución Política de Colombia, 1991, p. 182).

Con el pasar de los años, y teniendo como fundamento la Constitución de 1991, se realizaron varios cambios en lo referente a las funciones de los distintos niveles de gobierno y sus competencias, además de la disposición de recursos vía transferencias del gobierno central. Estas transformaciones y cambios en las responsabilidades de los diferentes niveles de gobierno se presentaron en las leyes 60 de 1993, 715 de 2001, y 1176 de 2007. En la siguiente Tabla 2 se podrá ver la asignación de los recursos en las diferentes leyes:

El proceso de descentralización en Colombia contó con gran éxito en el plano político, la escogencia vía voto popular de alcaldes y gobernadores representó un gran avance para la prestación eficiente de los bienes y servicios colectivos que la comunidad desea para su localidad. A pesar de este éxito, el proceso político dentro de la descentralización no está exento de dificultades, la corrupción de los procesos electorales son el principal reto que se presenta en este plano, si bien esta no es inherente al proceso de descentralización si no a la sociedad que lo converge, cabe mencionarlo como una amenaza.

Actualmente, la discusión sobre la descentralización en Colombia, encuentra su principal punto en torno a la sostenibilidad fiscal y la eficiencia en el manejo de los recursos por parte de los entes territoriales.

Analizando el modelo de Estado colombiano, es visible que es un modelo híbrido, ya que no cumple con las características propias del proceso de descentralización en el plano fiscal. Por un lado, el gobierno central otorga facultades a los entes territoriales para la cobranza de rentas que solventen o ayuden a estos para el ejercicio de sus funciones, y a su vez, contribuye por medio de transferencias con recursos para promover las políticas de orden nacional.

Aun cuando en teoría se presente una autonomía administrativa y política, la práctica evidencia una realidad completamente distinta, esto debido a que la planeación y el desarrollo de las políticas se encuentran regidas en buena medida por el gobierno central, que es quien decide en qué rublos y porcentajes se invierten los dineros que vía Sistema General de Participación (SGP) llegan a los entes territoriales.

Al mismo tiempo, los diferentes programas y agencias del gobierno central (como el Departamento de Prosperidad Social - DPS) cuentan con una presencia fuerte en las políticas municipales, lo que de una u otra forma hace que se desvirtúe la presencia de los gobernantes locales y sus políticas. Esto repercute en una clara pérdida de autonomía sobre las decisiones político-administrativas de los entes municipales, siendo esta presencia un refuerzo de la imagen del gobierno central como factor de poder en los entes territoriales. Una de las posturas más generalizadas sobre el proceso de descentralización en Colombia es la planteada por Jorge Asela y Sandra Hernández (2011), en la cual se evidencia los retrocesos en el proceso descentralización colombiano. El retroceso llega a tal punto, que se puede referir a un proceso de recentralización de recursos, argumentado por parte del gobierno central en la ineficiencia administrativa en el manejo de 
los recursos. Ejemplo claro es la aprobación de la Ley de Regalías y la creación del SGR (Sistema General de Regalías).

Una de las causantes de esta postura por parte del gobierno central podría deberse a la capacidad de absorción y de gestión de los entes territoriales, que actualmente se encuentra superada por el proceso de descentralización, lo que repercute en ineficiencia en la ejecución de las responsabilidades de los entes subnacionales. Es por ello que los diferentes niveles de gobierno han implementado políticas de fortalecimiento institucional a través de capacitaciones de nivel administrativo y gerencial, implementadas por las mismas territorialidades o los entes que las aglomeran (Federación Colombiana de Municipios, Federación Nacional de Departamentos). Este tipo de acciones le permiten a las territorialidades contar con mayores capacidades administrativas que puedan garantizar un uso más eficiente de los recursos.

En definitiva, el proceso de descentralización en Colombia, en los últimos 20 años, trajo consigo grandes contribuciones para el país en el plano político, avances en participación democrática con la elección de alcaldes y gobernadores, prestación de servicios públicos de mayor calidad y con amplia cobertura, veedurías ciudadanas como mecanismo de vigilancia sobre las acciones correspondientes a la gestión pública, son solo algunos de los aspectos positivos del proceso en Colombia. A su vez en términos de autonomía fiscal y administrativa, es otro el panorama: retrocesos en la asignación de recursos, pérdida de autonomía presupuestaría (Ley 715,1176 , Ley de Regalías) son algunas muestras del poder que aún ejerce el gobierno central sobre los entes territoriales y sus políticas.

Tabla 2. Asignación de recursos según Leyes 60 de 1993, 715 de 2001 y 1176 de 2007.

Table 2. Allocation of Resources According to Law 60 of 1993, 2001 and 11767152007

\begin{tabular}{|l|c|c|c|}
\hline \multicolumn{1}{|c|}{ Sectores } & Ley 60 de 1993 & $\begin{array}{c}\text { Ley } 715 \text { de } \\
2001\end{array}$ & $\begin{array}{c}\text { Ley } 1176 \text { de } \\
2007\end{array}$ \\
\hline Educación & $30 \%$ & $58,5 \%$ & $58,5 \%$ \\
\hline Salud & $25 \%$ & $24,5 \%$ & $24,5 \%$ \\
\hline Propósito general & - & $* \mathbf{1 7 \%}$ & $* \mathbf{1 1 , 6 \%}$ \\
\hline Agua potable y saneamiento básico & $\mathbf{2 0 \%}$ & $\mathbf{4 1 \%}$ & $5,4 \%$ \\
\hline Deporte, recreación y cultura & $5 \%$ & $\mathbf{7 \%}$ & \multirow{2}{*}{$\mathbf{8 3 \%}$} \\
\hline Fonpet & - & $\mathbf{1 0 \%}$ & \\
\hline Libre destinación & $\mathbf{2 0 \%}$ & $\mathbf{4 2 \%}$ & $\mathbf{1 7 \%}$ \\
\hline
\end{tabular}

Fuente: Elaborada por el autor con datos del DNP. La asignación de recursos de la Ley 715 de 2001 para los sectores de agua potable y saneamiento básico, deporte recreación y cultura, Fonpet y libre destinación, son derivados del total de recursos de propósito general (*17\%), del mismo modo la asignación de recursos en la Ley 1176 de 2007 para los sectores de deporte, recreación y cultura, Fonpet y libre destinación son derivados del total de los recursos de propósito general $(* 11,6 \%)$ según disposición de ley. 


\section{LA DESCENTRALIZACIÓN FISCAL VISTA DESDE EL INDICADOR DE DEPENDENCIA INTERGUBERNAMENTAL}

Para mostrar de manera más precisa cómo el proceso de descentralización en Colombia solo se ha dado políticamente, se preparó un análisis tomando como base el indicador de dependencia intergubernamental presentado por el Departamento Nacional de Planeación (DNP) para de una forma más clara, mostrar la carencia de los entes territoriales a la hora de financiar con recursos propios sus políticas, evidenciando el grado de dependencia de los mismos con el gobierno central y la ausencia de una descentralización fiscal.

El indicador de dependencia intergubernamental muestra el grado de incidencia del nivel central en los diferentes niveles de gobierno (departamentos, municipios). Esta incidencia es medida en relación con los ingresos recibidos por transferencias, más los dineros de regalía, como proporción de los ingresos totales de los entes territoriales. Lo cual se traduce en el grado de dependencia de los departamentos y municipios sobre los recursos transferidos por la nación para el financiamiento de los planes de desarrollo.

El indicador mide de 0 a 1 la incidencia de los recursos de transferencia en los ingresos totales de los municipios, siendo 1 el mayor grado de dependencia de este tipo de recursos. Para el análisis, se decidió crear tres categorías, i) No hay dependencia, que va desde 0 a 0,49, ii) Dependencia moderada, de 0,5 a 0,59 y iii) Dependencia mayoritaria de 0,6 a 1 . Se tomó como base para los municipios el año 2012 y para los departamentos los últimos 5 años de vigencia fiscal (2008-2012).

Revisando los resultados del análisis del indicador presentado por los municipios, se observa que de los 1101 municipios, 913 cuentan con una dependencia mayoritaria de los recursos provenientes del gobierno central para el ejercicio de sus funciones y el cumplimiento de sus obligaciones; 84 cuentan con una dependencia moderada y tan solo 104 municipios no cuentan con dependencia de los recursos de la nación (ver Fig. 1). Que el $82,92 \%$ de los municipios de un país cuenten con mayoritaria dependencia de los recursos transferidos por la nación para ejercer sus funciones y que solo el $9,45 \%$ de los mismos no sea dependiente de este tipo de recursos, evidencia que el proceso de descentralización fiscal en Colombia aún está muy lejano de ser una realidad, y mientras no se avance en este campo, los municipios no podrán cumplir con eficiencia y eficacia las funciones y competencias consagradas por la ley (Ley 60 de 1993).

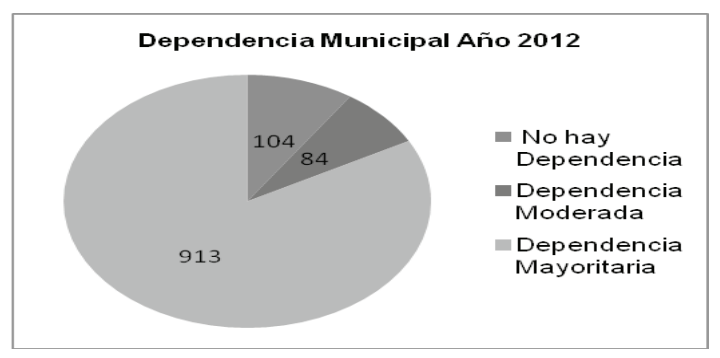

Figura 1. Dependencia municipal para el año 2012 Figure 1. Municipal Unit for 2012

Fuente: Elaborado por el autor con datos tomados del DNP

En el caso de los departamentos las cosas no son muy diferentes a lo evidenciado anteriormente para los municipios. De los 32 departamentos en el promedio de los últimos 5 años (2008-2012), 21 cuentan con dependencia mayoritaria y 5 con dependencia moderada para financiar sus planes de desarrollo; 6 departamentos no dependen en gran medida de los recursos de la nación para desarrollar sus funciones y competencias (ver Fig. 2). 


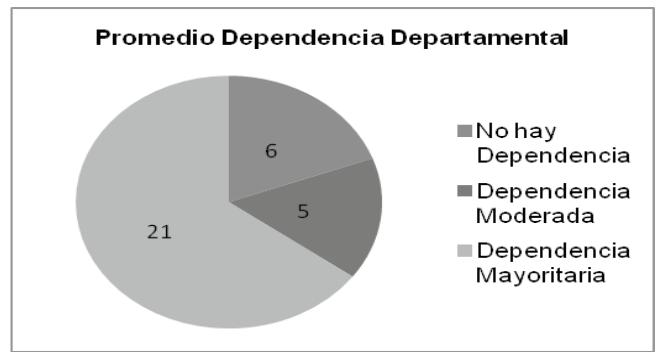

Figura 2. Promedio dependencia departamental últimos 5 años (2008-2012)

Figure 2. Average Departmental Agency Last 5 Years (2008-2012)

Fuente: Elaborado por el autor con datos del DNP
En el transcurso de esos 5 años, solo 4 departamentos no generaron dependencia de los recursos que vía transferencias y regalías entrega el gobierno central, (Antioquia, Atlántico, Cundinamarca y Valle del Cauca). Departamentos como Risaralda, San Andrés, Santander, Quindío y Caldas cuentan con una moderada dependencia, siendo Risaralda el departamento más cercano a salir de dicha posición, lo cual se puede ver en el siguiente Tabla 3.

Tabla 3. Departamentos sin o moderada dependencia intergubernamental (2008-2012)

Table 3. Departments without moderate or intergovernmental agencies (2008-2012)

\begin{tabular}{|c|c|c|c|c|c|}
\hline & \multicolumn{5}{|c|}{ AÑO } \\
\hline Departamentos & $\mathbf{2 0 0 8}$ & $\mathbf{2 0 0 9}$ & $\mathbf{2 0 1 0}$ & $\mathbf{2 0 1 1}$ & $\mathbf{2 0 1 2}$ \\
\hline Antioquia & 0,387 & 0,395 & 0,385 & 0,405 & 0,326 \\
\hline Atlántico & 0,403 & 0,424 & 0,420 & 0,445 & 0,368 \\
\hline Cundinamarca & 0,360 & 0,377 & 0,372 & 0,415 & 0,346 \\
\hline Valle Del Cauca & 0,428 & 0,455 & 0,435 & 0,492 & 0,421 \\
\hline Risaralda & 0,507 & 0,495 & 0,518 & 0,515 & 0,449 \\
\hline San Andrés & 0,572 & 0,562 & 0,569 & 0,511 & 0,469 \\
\hline Santander & 0,518 & 0,620 & 0,590 & 0,528 & 0,354 \\
\hline Quindío & 0,524 & 0,570 & 0,556 & 0,545 & 0,541 \\
\hline Caldas & 0,582 & 0,526 & 0,539 & 0,455 & 0,528 \\
\hline
\end{tabular}

Fuente: Elaborado por el autor con datos del DNP

Tanto a nivel departamental como municipal, la dependencia financiera de los entes territoriales con respecto al gobierno central es elevada, lo cual ratifica la tesis sobre que la descentralización en Colombia solo se ha presentado en lo correspondiente a lo político, ya que en lo fiscal los entes territoriales son altamente dependientes de las transferencias del ente central, y lo más preocupante es que no se observa un panorama claro que muestre que se están realizando los ajustes o cambios necesarios para darle vuelta a esa dependencia.

\section{CONCLUSIONES}

El proceso de descentralización se constituye como el modelo más idóneo para la estructuración del Estado moderno, debido a factores como la lectura de la revelación de las preferencias de los individuos, una mayor eficiencia administrativa y una vinculación más cercana de la ciudadanía al accionar público.

La Constitución Política de 1991 se constituye como el marco legal que afianza el proceso de descentralización en Colombia, sus avances a nivel político, administrativo y 
fiscal representaron un punto de partida idóneo para consolidar el proceso.

La descentralización en Colombia debe ser entendida como un proceso complejo que reúna las diferentes dinámicas, tanto sociales, económicas y culturales. Por ello debe ser influenciado y enriquecido por las diferentes visiones que componen la realidad Colombiana.

Las transferencias del gobierno central no deben ser entendidas por este como una forma de fortalecer su presencia y poder en los entes territoriales sino como algo más profundo, buscando sinergia entre las políticas nacionales y las necesidades de las comunidades.

El proceso de descentralización en Colombia es una realidad en cuanto a su concepción como marco institucional, cuenta con grandes avances a nivel político, la elección a través del voto popular de gobernadores, alcaldes y sus correspondientes órganos colegiados (concejos, asambleas) son sin ninguna duda aspectos sobresalientes dentro del proceso de descentralización, pero en el plano fiscal y de autonomía, la mayoría de los entes territoriales están limitados, lo que impide un correcto funcionamiento $y$ cumplimiento de sus competencias.

\section{REFERENCIAS}

Asela, J. E. y Hernández, S. M. (2011). Dinámica fiscal en Barrancabermeja: el papel de las regalías petroleras (2000-2010). (Tesis de pregrado). Universidad Industrial de Santander. Bucaramanga, Colombia.

Constitución Política de Colombia (2012). Bogotá: Legis.
Departamento Nacional De Planeación. Recuperado de: https://www.dnp.gov.co/programas/ desarrollo-territorial/evaluacion-yseguimiento-de-ladescentralizacion/Paginas/evaluacio $\mathrm{n}$-y-seguimiento-de-ladescentralizacion.aspx

Finot, I. (2001). Descentralización en América latina: Teoría y práctica. Recuperado de:

http://www.eclac.org/publicaciones/ xml/9/7259/lcl1521e.pdf

Hirschman, A. (1970). Exit, Voice, and Loyalty. Cambridge: Harvard University Press.

Lleras, C. y Posada, J. (1995). Descentralización política fiscal y administrativa después de la constitución de 1991. Bogotá: Fundación Presencia.

Musgrave, R. (1959). The Theory of Public Finance. Tokio: McGraw-Hill.

Restrepo, J. (2001). Hacienda Pública. Bogotá: Universidad Externado de Colombia.

Ruiz, F. (2000). Evolución del proceso de descentralización fiscal en el municipio de Bucaramanga (19841997). (Tesis de pregrado). Universidad Industrial de Santander. Bucaramanga, Colombia.

Samuelson, P. (1954). A Pure Theory of Local Expenditures. Review of Economics and Statistics, 36(4), 387-389.

Tiebout, C. (1956). A Pure Theory of Local Expenditures. Journal of Political Economy, 64(5), 416-424. 